\title{
Determinantes do compartilhamento do conhecimento visando a cooperação internacional em ciência e tecnologia no Brasil
}

Fernanda Cristina Barbosa Pereira Queiroz

Professora do departamento de Engenharia de Produção da UFRN. Pós-doutora em Ciência, Gestão e Tecnologia da Informação pela UFPR. Mestre e doutora em Engenharia de Produção pela UFSC. Graduada em Ciências Econômicas pela UFJF.

Helena de Fátima Nunes Silva

Professora do Programa de Pós-Graduação em Ciência, Gestão e Tecnologia da Informação, da UFPR. Doutora em Engenharia de Produção pela UFSC. Mestre em Educação pela UFPR. Graduada em Biblioteconomia pela UFSC.

Paulo Henrique de Almeida

\begin{abstract}
Mestrando do Programa de Pós-Graduação em Ciência, Gestão e Tecnologia da Informação da UFPR. Especialista em Gestão Pública pela Faculdades de Administração, Ciências, Educação e Letras - FACEL. Graduado em Administração. Administrador no Instituto Federal do Paraná IFPR.
\end{abstract}

http://dx.doi.org/10.1590/1981-5344/2748

Este trabalho tem como objetivo identificar os fatores que influenciam o compartilhamento do conhecimento entre os Pesquisadores Visitantes Especiais (PVE) e os pesquisadores vinculados às Instituições de Ensino $e$ Pesquisa (IEP) localizadas no país. A pesquisa consistiu na identificação dos proponentes dos projetos de pesquisa aprovados nas chamadas realizadas em 2013 pelo CNPq por meio do Programa Ciência Sem Fronteiras. A coleta de dados foi realizada junto aos pesquisadores das IEP que receberam os pesquisadores estrangeiros, por meio de.um questionário on-line. A análise foi feita por meio da estatística descritiva e análise fatorial exploratória e confirmatória. Os resultados encontrados permitiram a categorização de cinco fatores que influenciam o compartilhamento do conhecimento: Organização 
Receptora, Redes e Recompensas, Características do Transmissor, Gestão e Tecnologia.

Palavras-chave: Compartilhamento de conhecimento; Programa Ciência Sem Fronteiras; Cooperação internacional.

\title{
Determining the knowledge sharing aiming at international cooperation in science and technology in Brazil
}

\begin{abstract}
This aim of this work is to identify the factors influencing knowledge sharing among Special Visiting Researchers (SVR) and the researchers of the institutions of Education and Research located in Brazil. The research was the identification of host research projects approved in calls made in 2013 by CNPq through the program Science Without Borders. Data collection through online questionnaire was conducted with host researchers from Brazil that receiving foreign researchers. The analysis was done using descriptive statistics and exploratory and confirmatory factor analysis. The results allowed the categorization of five factors that influence knowledge sharing Organization Receiving, Networks and Rewards, Transmitter Features, Management and Technology
\end{abstract}

Keywords: Knowledge sharing; Science Without Borders Program; International cooperation.

Recebido em 28.03.2016 Aceito em26.09.2016

\section{Introdução}

O presente estudo discute os fatores determinantes para o compartilhamento do conhecimento em equipes de pesquisadores brasileiros e estrangeiros consideradas de excelência. Sato, Silva e Drago (2013), com base em Nonaka e Takeuchi (1997) colocam que o processo de criação do conhecimento organizacional é composto das seguintes fases: compartilhamento do conhecimento tácito; criação de novos conceitos; justificação de conceitos; construção de arquétipo ou protótipo; difusão interativa do conhecimento. Após o reconhecimento da importância da criação do conhecimento organizacional é necessário que este conhecimento seja compartilhado para que se gerem inovações (SATO, SILVA; DRAGO, 2013). 
O compartilhamento do conhecimento envolve a comunicação entre as pessoas. Contudo, mesmo sabendo que a tecnologia é útil na comunicação do conhecimento explícito é fundamental ressaltar que a comunicação do conhecimento intrínseco e a criação de novos conhecimentos exigem a interação social e a participação humana (AJMAL; KOSKINEN, 2007).

Cyr e Choo (2010) conceituam o compartilhamento de conhecimento como uma forma de interação social em que é moderada pela orientação de valor social do indivíduo. No sentido de facilitar a interação social, a cooperação pode ser entendida como uma ação social articulada, alinhavada por objetivos comuns visando a solução de problemas concretos (BRUNI, 2005).

Os programas de cooperação internacional são criados inicialmente como estratégia de formação de pessoal qualificado, contudo os pesquisadores no decorrer de sua vida acadêmica, estabelecem laços não só de consumo de bibliografia, mas de relações acadêmicas possibilitando, desta forma, a produção de conhecimento por meio de projetos de pesquisas conjuntos (WESTPHAL, 2014).

No contexto da cooperação internacional, o Programa Ciência Sem Fronteiras (Brasil) concede apoio financeiro a projetos de pesquisa que visem, por meio do intercâmbio, da mobilidade internacional e da cooperação científica e tecnológica, promover a consolidação, expansão e internacionalização da ciência e tecnologia, da inovação e da competitividade do Brasil com enfoque nas áreas consideradas prioritárias (BRASIL, 2011).

Em relação à atração de pesquisadores, o Programa Ciência Sem Fronteiras atraiu, até 2015, cerca de 2000 Pesquisadores Visitantes Especiais (PVE), ou seja, a atração de grandes lideranças científicas residentes no exterior. 'É neste ponto que se formula a questão que orienta esta pesquisa: quais os fatores que influenciam 0 compartilhamento de informações e conhecimento entre os pesquisadores estrangeiros e brasileiros vinculados às Instituições de Ensino e Pesquisa nacionais? Diante destas considerações, o objetivo da presente pesquisa é identificar quais fatores influenciam no compartilhamento de informações e conhecimentos entre os Pesquisadores Visitantes Especiais (PVE) e os pesquisadores vinculados às Instituições de Ensino e Pesquisa localizadas no Brasil.

Como justificativa teórica para o presente estudo, é importante destacar que o compartilhamento do conhecimento entre pesquisadores permite a criação de novos conhecimentos e a geração de inovações. O tema compartilhamento de conhecimento é recente, sendo que as publicações começaram a surgir de modo mais expressivo nas áreas de Ciências da Informação e Administração a partir de 2009 (OSINSKI; ROMAN; SELIG, 2015).

Associado ao fato de que as pesquisas sobre mobilidade acadêmica internacional com a atração de pesquisadores ao Brasil são raras, tendo em vista que o país tradicionalmente enviou seus pesquisadores ao 
exterior para capacitação e treinamento. Cabe ressaltar como reforço a esta justificativa o fato de que o momento de atração de cientistas de maneira planejada e desconcentrada geograficamente teve início com o Programa Ciência Sem Fronteiras, uma vez que experiências anteriores eram concentradas em centros de excelência no país (FUNDAÇÃO DE AMPARO À PESQUISA DO ESTADO DE SÃO PAULO, 2010). Acredita-se que o presente artigo contribui com os estudos referentes ao Programa e seus desdobramentos.

\section{Fatores que influenciam o compartilhamento do conhecimento}

A cooperação científica e tecnológica internacional remete a um esforço organizado, envolvendo dois ou mais países, com o objetivo do intercâmbio de conhecimento, métodos e processos científicos, possibilitando inclusive a transferência de tecnologia para geração de inovações tecnológicas (DUARTE, 2008)

O conhecimento passou a ser um insumo importante no processo inovativo e a sua criação interna, por parte das empresas, vem se tornando a principal fonte de competitividade. Neste contexto, o papel das universidades vem adquirindo maior relevância, uma vez que ainda se apresentam como locus principal de geração de novos conhecimentos (MANSFIELD, 1991; RAPINI; RIGHI, 2005; KNOCKAERT et al., 2011).

A geração de novos conhecimentos depende especialmente do compartilhamento. Este ocorre tanto de modo formal, por meio de patentes e acordos de royaties, como informal, pela interação e colaboração entre pesquisadores e profissionais da indústria. As dificuldades encontradas pelos pesquisadores para o desenvolvimento de uma pesquisa possibilitam a criação e/ou fortalecimento da relação com seus pares (ALCARA et al., 2009).

Para Bousari e Hassanzadeh (2012) sem o compartilhamento, a missão da produção científica, que se baseia na produção de conhecimento científico, não será concretizada; por outro lado, cooperações de pesquisa têm efeito significativo no aumento de produções científicas e suas citações. Existem na literatura algumas perspectivas distintas para analisar os fatores que influenciam o compartilhamento do conhecimento. Contudo, apesar das diferenças na categorização dos grupos ou dimensões, observa-se que os fatores são analisados de modo convergente pela ótica do indivíduo, da organização ou do ambiente (DELGADO et al., 2013).

As organizações ainda carecem de estudos empíricos que investiguem como as características individuais e organizacionais influenciam o compartilhamento do conhecimento (WANG; NOE; WANG, 2011). Esta visão é compartilhada por Tohidinia e Mosakhani (2010) que acreditam que os fatores que facilitam ou dificultam este processo ainda não foram propriamente identificados. 
Os fatores que influenciam o compartilhamento do conhecimento entre indivíduos são a natureza do conhecimento, a motivação para compartilhar, as oportunidades para compartilhar e a cultura do ambiente de trabalho. O compartilhamento do conhecimento entre vários indivíduos com diferentes origens, perspectivas e motivações torna-se um passo fundamental para a criação da aprendizagem organizacional (IPE, 2003)

Alguns autores agruparam as barreiras ao compartilhamento do conhecimento por meio de uma tríade: individual, organizacional e tecnológica (CHASE, 1997; DE LONG; FAHEY, 2000; RIEGE, 2005). É possível analisar os fatores facilitadores utilizando a mesma categorização (NODARI et al., 2012).

No nível individual os obstáculos e facilitadores ao compartilhamento do conhecimento estão relacionados àqueles de foro íntimo como expectativas, valores, atitudes, percepção, personalidade, emoções, sentimentos, disposição e motivação.

No nível organizacional os fatores estão relacionados à estratégia, modelo de gestão, estrutura organizacional, infraestrutura e leiaute, tamanho das unidades de negócios, liderança, aos processos operacionais, à cultura, aos sistemas de recompensas (PAGHALED; SHAFIEZADEH; MOHAMMADI, 2011).

No nível tecnológico os fatores de influência ocorrem por intermédio dos processos de tecnologia da informação e uso dos sistemas de informação. (PAGHALED; SHAFIEZADEH; MOHAMMADI, 2011).

Para $\mathrm{Hsu}^{1}$ (2006 apud DELGADO et al., 2013) existem três enfoques para se analisar o compartilhamento do conhecimentos: o enfoque baseado nas ferramentas, o enfoque baseado nos incentivos e o enfoque integrador.

O enfoque baseado nas ferramentas centra a discussão no uso da Tecnologia da Informação para o compartilhamento do conhecimento (KIM; LEE, 2006; TOHIDINIA; MOSAKHANI, 2010). A segunda visão tem como princípio a racionalidade, pois se acredita que os funcionários se dispõem a compartilhar o conhecimento caso haja suporte gerencial que os estimulem por intermédio de recompensas. Este enfoque é centrado no uso de incentivos para promover o compartilhamento do conhecimentos (WOLFE; LORASS; 2008). E o enfoque integrador compreende os fatores sociais, sejam estes individuais ou organizacionais (IPE, 2003; ORDAZ; CRUZ; GINEL, 2010).

Wang e Noe (2010), ao revisarem a literatura a respeito dos fatores que influenciam o compartilhamento do conhecimento, identificaram questões emergentes e a necessidade de investigações futuras. Na visão dos autores, existem cinco áreas que influenciam o compartilhamento de conhecimento, sendo que o contexto organizacional, as características interpessoais e da equipe, as características culturais e as características individuais influenciam direta ou indiretamente os fatores motivacionais.

\footnotetext{
${ }^{1} \mathrm{HSU}, \mathrm{I}$. Enhancing employee tendencies to share knowledge-Case studies on nine companies in Taiwan. International Journal of Information Management, v. 26, n. 4, p. 326-338, 2006
} 


\subsection{Fatores individuais ou da equipe}

Conforme Riege (2005), a capacidade das pessoas para compartilhar conhecimento depende, em primeiro lugar, das suas habilidades de comunicação. Uma comunicação eficaz, tanto verbal como escrita, é fundamental para a partilha de conhecimentos (DAVENPORT; PRUSAK, 1998).

Alguns estudos destacam as redes sociais e a capacidade das pessoas interagirem como facilitadores para o compartilhamento do conhecimento (BARON; MARKMAN, 2000; INGRAM; BAUM , 1997; NAHAPIET; GHOSHAL, 1998). Cho, Li e Su (2007) destacam que as interações sociais anteriores ao processo, a formação e o desenvolvimento de comunidades, o feedback coletivo e individual e a cooperação interpessoal são mecanismos que facilitam o compartilhamento.

Os fatores individuais são decisivos para o compartilhamento do conhecimento, sendo que na maior parte dos casos os trabalhadores são quem efetivamente decidem se querem compartilhar ou não seus conhecimentos (DUGUID, 2005). Conforme Holste e Fields (2010), apesar da tecnologia facilitar o armazenamento do conhecimento explícito, o conhecimento tácito reside nas mentes das pessoas e sua disponibilidade e uso dependem das relações e decisões individuais.

Phillips et al. (2004) enfatizam a similaridade, a familiaridade e a simpatia como motivadores para as pessoas compartilharem informações dentro de um grupo. As relações de confiança também influenciam o compartilhamento do conhecimento. Assim, a qualidade do relacionamento entre colegas de trabalho associada à percepção de competência e profissionalismo contribuem para que alguém esteja disposto a compartilhar (LUCAS, 2005; HOLSTE; FIELDS, 2010; ALCARA et al., 2009,; KUO, 2013; HO; KUO; LIN, 2012).

A falta de confiança mútua; as diferentes culturas, vocabulários e quadros de referência; a falta de tempo e de lugar de encontro, ideia estreita de trabalho produtivo; o fato do status e recompensas irem para os possuidores de conhecimento; a falta de capacidade de absorção pelos recipientes; a crença de que o conhecimento é prerrogativa de determinados grupos e a intolerância com erros ou necessidade de ajuda são barreiras que dificultam o compartilhamento do conhecimento (DAVENPORT; PRUSAK, 1998).

Ainda no nível individual, os obstáculos são aqueles que envolvem a capacidade que cada pessoa tem para lidar com novas situações, eventos, informações e contextos (LIN; LEE, 2006; ALCARA et al., 2009; HONG; SUH; KOO, 2011).

Especificamente no caso de ambiente de pesquisa e desenvolvimento, de acordo com Ensign (2008) e Ensign e Hebert (2010), um item que pode facilitar ou retardar o compartilhamento voluntário de conhecimentos é a reputação do receptor. Assim sendo, tanto o comportamento passado do receptor como as expectativas de ações no futuro influenciam o transmissor do conhecimento a compartilhar ou não. 


\subsection{Fatores organizacionais e tecnológicos}

O compartilhamento do conhecimento entre os indivíduos de uma organização não pode ser forçado, mas pode ser encorajado pelas políticas organizacionais e facilitado pela estrutura organizacional (KUO, 2013).

Mayo ${ }^{2}$ (2003 apud CARVALHO; MENDES; VERAS, 2006, p. 133), afirma que cinco fatores são fundamentais na motivação para o compartilhamento do conhecimento: (i) direção de liderança e estilo de gerenciamento; (ii) ambiente físico; (iii) grupo de trabalho; (iv) oportunidades para aprender $e(v)$ desenvolver $e$ recompensa e reconhecimento. Para Tohidinia e Mosakhani (2010), outra variável importante é o clima organizacional. Sendo que algumas organizações concedem recompensas e facilidades tecnológicas para seus funcionários objetivando estimular o compartilhamento do conhecimento.

Para Stewart (1998), o estímulo ao compartilhamento, a motivação das pessoas para a cooperação, o comportamento colaborativo e a relação de confiança pertencem aos componentes da cultura organizacional. Assim, de acordo com Jo e Joo (2011), o compartilhamento do conhecimento requer uma norma adequada da organização (cultura organizacional), uma sensação de identificação (comprometimento organizacional) e altruísmo voluntário (comportamento de cidadania organizacional). De acordo com Kim e Lee (2006), tanto no setor público como no setor privado, as percepções dos funcionários em relação à centralização da organização afetam a disponibilidade dos mesmos em compartilhar informações e conhecimentos.

Os obstáculos organizacionais ao compartilhamento do conhecimento destacados por Floriano (2010) são a distância física; a estrutura organizacional; o sistema de recompensas da organização, o status, a desconfiança, desconhecimento e o poder. Wolfe e Lorass (2008) acreditam que os funcionários estão dispostos a compartilhar 0 conhecimento se houver suporte gerencial que os estimulem por meio de recompensas.

O uso da Tecnologia da Informação (TICs) permite uma busca mais rápida e acesso à informação e conhecimento. (KIM; LEE, 2006; LIN, 2007; TOHIDINIA; MOSAKHANI; 2010). As TICs permitem que as organizações expandam as redes sociais e criem colaboração efetiva. Desta forma, as TICs melhoram a comunicação entre os especialistas, aproximam virtualmente as pessoas e promovem 0 intercâmbio. (DELGADO et al., 2013).

Nem todos os ambientes são adequados ao compartilhamento e criação de conhecimento, por enfrentarem barreiras individuais e organizacionais. Portanto, quanto melhor forem as relações entre as pessoas maior será o compartilhamento do conhecimento. Davenport e Prusak (1998) afirmam que o compartilhamento do conhecimento ocorre

\footnotetext{
2 MAYO, A. O valor humano da empresa: valorização das pessoas como ativos. São Paulo: Prentice Hall, 2003.
} 
de maneira informal, ou seja, espontânea e não estruturada, como, por exemplo, conversas de corredores e paradas para cafezinho; ou de maneira formal, por meios estruturados.

\section{Encaminhamento Metodológico}

Esta pesquisa utilizou uma abordagem quantitativa de natureza exploratória-descritiva. Quanto aos objetivos foi utilizada a pesquisa descritiva precedida da pesquisa exploratória e da pesquisa bibliográfica, pois no primeiro momento buscou-se o levantamento e o conhecimento das variáveis pertinentes aos pesquisadores visitantes internacionais e os professores brasileiros pesquisados.

Foram pesquisados os proponentes dos projetos aprovados na chamada 71/2013 (Linha 2, Calendários 1, 2 e 3) realizada pelo Conselho Nacional de Pesquisa (CNPq). Os proponentes são pesquisadores vinculados a alguma instituição de pesquisa no Brasil que recebeu os pesquisadores estrangeiros. Dos 339 projetos aprovados, 29 não tinham iniciado a pesquisa até a aplicação do questionário, portanto a população alvo foi de 310 proponentes.

$O$ instrumento de coleta de dados foi elaborado considerando 15 variáveis que influenciam o compartilhamento do conhecimento, mensuradas por uma escala Likert de 5 pontos. O questionário foi encaminhado aos proponentes utilizando o software Qualtrycs. A amostra foi de 121 proponentes que responderam o questionário e os dados foram analisados por meio da estatística descritiva. Utilizou-se a Análise Fatorial Exploratória (AFE) por meio do software SPSS 21 e Análise Fatorial Confirmatória (AFC) utilizando o software SmartPLS 3.2.

A estatística descritiva foi utilizada para se calcular a média, mediana e desvio padrão das variáveis analisadas. A AFE foi empregada para examinar o relacionamento de um grande número de variáveis e determinar se essa informação poderia ser condensada ou sumarizada em um conjunto reduzido de fatores. A AFC foi utilizada para se avaliar se os itens possuíam validade e confiabilidade, aqueles que apresentaram baixas cargas fatoriais (falta de validade convergente e confiabilidade) foram excluídos da escala, assim como os itens que tinham alta carga cruzada, o que indica falta de validade discriminante (HAIR JR. et al., 2014).

\section{Resultados encontrados}

Nesta seção são apresentados os resultados e análises dos dados.

\subsection{O Programa Ciência Sem Fronteiras e a atração de pesquisadores estrangeiros}

O programa Ciência sem Fronteiras, regulamentado pelo Decreto 7.642/2011, é uma parceria entre o Ministério da Ciência e Tecnologia e o Ministério da Educação, por meio de suas respectivas instituições de 
fomento (CNPq) e a Comissão de Aperfeiçoamento de Pessoal do Nível Superior (CAPES) e tem por objetivo promover de maneira acelerada 0 desenvolvimento tecnológico e estimular os processos de inovação no Brasil por meio da promoção da mobilidade internacional de docente, discente de graduação e pós-graduação, de pós-doutorandos brasileiros e pesquisadores brasileiros e estrangeiros, estimulando a inserção das pesquisas feitas nas instituições brasileiras às melhores experiências internacionais.

Em 2013, na chamada $71 / 2013$ realizada em três (3) etapas foram aprovados 339 pesquisadores na Linha 2. A maior parte dos pesquisadores visitantes realizou suas atividades no Estado de São Paulo (23\%), seguido pelos estados do Rio de Janeiro (18\%), Rio Grande do Sul (11\%), Santa Catarina (7\%) e Minas Gerais (7\%).

As áreas de pesquisa mais procuradas pelos PVE foram Engenharias (15\%), Ciências Exatas e da Terra - Física e Matemática (15\%) seguidas das áreas Biodiversidade e Bioprospecção (10\%), Biologia e Ciências Biomédicas (10\%) e Biotecnologia (10\%).

Os Estados Unidos enviou 68 pesquisadores internacionais ao Brasil, seguido da Espanha que enviou 45 pesquisadores, França (40 pesquisadores) e Portugal (31 pesquisadores). Contudo, cabe destacar a presença de pesquisadores de aproximadamente 50 países diferentes da América, Europa, África, Oceania e Ásia. Essa distribuição de pesquisadores de diferentes países sinaliza o potencial brasileiro para atrair lideranças internacionais de todo mundo.

\subsection{Caracterização do perfil dos respondentes}

Os resultados encontrados evidenciam que $88 \%$ dos respondentes possuem mais de 41 anos e $84 \%$ fizeram parte da graduação ou pósgraduação no exterior. Dentre os motivos mais citados para a atração de um Pesquisador Visitante Especial para o Brasil estão a possibilidade de realizar pesquisas $(93 \%)$ e publicar em conjunto $(83 \%)$, conforme Tabela 1.

Tabela 1 - Motivos para a atração do PVE: chamada 71/2013

\begin{tabular}{l|c|c}
\hline \multicolumn{1}{c|}{ Motivos } & Respostas & \% \\
\hline \hline Realização de pesquisas & 112 & $93 \%$ \\
\hline Publicação em conjunto & 101 & $83 \%$ \\
\hline Aprendizado da equipe & 82 & $68 \%$ \\
\hline $\begin{array}{l}\text { Internacionalização da } \\
\text { ciência }\end{array}$ & 73 & $60 \%$ \\
\hline Geração de inovações & 49 & $40 \%$ \\
\hline Outros & 5 & $4 \%$ \\
\hline
\end{tabular}

Observação: respostas múltiplas.

Fonte: Dados da pesquisa (2015). 
Determinantes do compartilhamento do conhecimento visando a cooperação internacional em ciência e tecnologia no Brasil
Fernanda Cristina Barbosa Pereira Queiroz; Helena de Fátima Nunes Silva; Paulo Henrique de Almeida

De um modo geral, os proponentes conheceram os PVE no desenvolvimento de projetos anteriores (61\%) e eventos que participaram (31\%), o que sinaliza o amadurecimento das relações. Apenas $10 \%$ dos respondentes não conheciam pessoalmente o PVE e 7\% o conhecia apenas pela literatura (Tabela 2)

Tabela 2 - Origem do relacionamento: proponente e PVE

\begin{tabular}{l|c|c}
\hline \multicolumn{1}{c|}{ Onde conheceu o pesquisador } & Respostas & \% \\
\hline \hline No desenvolvimento de projetos anteriores & 74 & $61 \%$ \\
\hline Em eventos & 38 & $31 \%$ \\
\hline Não o conhecia pessoalmente & 12 & $10 \%$ \\
\hline Somente pela literature & 9 & $7 \%$ \\
\hline Orientação de pós-graduação & 6 & $5 \%$ \\
\hline Outros: & 5 & $4 \%$ \\
\hline
\end{tabular}

Observação: respostas múltiplas.

Fonte: Dados da pesquisa (2015)..

A competência do pesquisador visitante especial, seja na área de atuação do proponente (74\%) ou em área complementar (51\%), é o principal motivo para que o proponente convidasse o PVE.

\subsection{Fatores facilitadores e inibidores ao compartilhamento de informações e conhecimento}

A confiança, a tecnologia da informação (internet e sistemas de informação) experiência e habilidade de comunicação do pesquisador estrangeiro são variáveis determinantes que favorecem 0 compartilhamento do conhecimento, apresentando as maiores médias conforme ilustra a Tabela 3.

Tabela 3 - Variáveis que influenciam o compartilhamento: chamada $71 / 2013$

\begin{tabular}{l|c|c|c}
\hline \multicolumn{1}{c|}{ Variáveis } & Média & $\begin{array}{c}\text { Desvio } \\
\text { Padrão }\end{array}$ & Mediana \\
\hline $\begin{array}{l}\text { A confiança facilita o compartilhamento de } \\
\text { conhecimentos entre o Pesquisador Visitante } \\
\text { Especial e a equipe brasileira (Variavel P3) }\end{array}$ & 4.74 & 0.50 & 5 \\
\hline $\begin{array}{l}\text { A Internet facilita o compartilhamento de } \\
\text { conhecimento entre o Pesquisador Visitante } \\
\text { Especial com a equipe brasileira (Variavel T1) }\end{array}$ & 4.73 & 0.50 & 5 \\
\hline $\begin{array}{l}\text { O nível elevado de experiência do Pesquisador } \\
\text { Visitante Especial facilita o compartilhamento de } \\
\text { conhecimento (Variavel P4) }\end{array}$ & 4.66 & 0.56 & 5 \\
\hline $\begin{array}{l}\text { A habilidade de comunicação do Pesquisador } \\
\text { Visitante Especial facilita o compartilhamento de } \\
\text { conhecimentos (Variavel P1) }\end{array}$ & 4.46 & 0.73 & 5 \\
\hline $\begin{array}{l}\text { Os sistemas de informação facilitam o } \\
\text { compartilhamento de conhecimento entre o }\end{array}$ & 4.45 & 0.80 & 5 \\
\hline
\end{tabular}




\begin{tabular}{|c|c|c|c|}
\hline $\begin{array}{l}\text { Pesquisador Visitante Especial e a equipe } \\
\text { brasileira (Variavel T2) }\end{array}$ & & & \\
\hline $\begin{array}{l}\text { As oportunidades de publicações em periódico de } \\
\text { qualidade facilitam o compartilhamento de } \\
\text { conhecimento entre o Pesquisador Visitante } \\
\text { Especial e a equipe brasileira(Variavel P2) }\end{array}$ & 4.31 & 0.77 & 4 \\
\hline $\begin{array}{l}\text { O clima organizacional (percepção coletiva que as } \\
\text { pessoas têm do ambiente de trabalho) facilitou o } \\
\text { compartilhamento de conhecimentos do } \\
\text { Pesquisador Visitante Especial com a equipe } \\
\text { brasileira (Variavel O2) }\end{array}$ & 3.88 & 1.03 & 4 \\
\hline $\begin{array}{l}\text { A infraestrutura da Universidade/Centro de } \\
\text { pesquisa brasileiro facilitou o compartilhamento } \\
\text { de conhecimentos do Pesquisador Visitante } \\
\text { Especial com a equipe brasileira (Variavel O1) }\end{array}$ & 3.79 & 1.05 & 4 \\
\hline $\begin{array}{l}\text { As redes de relacionamento e de cooperação } \\
\text { facilitam o compartilhamento de conhecimento } \\
\text { entre o Pesquisador Visitante Especial e a equipe } \\
\text { brasileira (Variavel P5) }\end{array}$ & 3.69 & 1.18 & 4 \\
\hline $\begin{array}{l}\text { A estrutura organizacional da Universidade/Centro } \\
\text { de Pesquisa facilitou o compartilhamento de } \\
\text { conhecimentos do Pesquisador Visitante Especial } \\
\text { com a equipe brasileira (Variavel O4) }\end{array}$ & 3.59 & 1.16 & 4 \\
\hline $\begin{array}{l}\text { A cultura organizacional (crenças, valores) } \\
\text { facilitou o compartilhamento de conhecimentos do } \\
\text { Pesquisador Visitante Especial com a equipe } \\
\text { brasileira (Variavel O3) }\end{array}$ & 3.35 & 1.30 & 3 \\
\hline $\begin{array}{l}\text { Os Líderes e gestores da sua Universidade /Centro } \\
\text { de Pesquisa facilitaram para que ocorresse o } \\
\text { compartilhamento de conhecimentos entre o } \\
\text { Pesquisador Visitante Especial e a equipe } \\
\text { brasileira (Variavel O6) }\end{array}$ & 3.23 & 1.46 & 3 \\
\hline $\begin{array}{l}\text { As Recompensas e Incentivos facilitam o } \\
\text { compartilhamento de conhecimento entre o } \\
\text { Pesquisador Visitante Especial e a equipe } \\
\text { brasileira (Variavel O7) }\end{array}$ & 3.20 & 1.29 & 3 \\
\hline $\begin{array}{l}\text { A alta direção da Universidade / Centro de } \\
\text { Pesquisa facilitou o compartilhamento de } \\
\text { conhecimentos entre o Pesquisador Visitante } \\
\text { Especial e a equipe brasileira (Variavel O5) }\end{array}$ & 3.11 & 1.49 & 3 \\
\hline $\begin{array}{l}\text { As redes sociais e aplicativos (facebook, } \\
\text { WhatsApp etc) facilitam o compartilhamento de } \\
\text { conhecimento entre o Pesquisador Visitante } \\
\text { Especial e a equipe brasileira (Variavel T3) }\end{array}$ & 2.75 & 1.32 & 3 \\
\hline
\end{tabular}

Fonte: Dados da pesquisa (2015).

Para verificar a aplicação da Análise Fatorial (AF) utilizou-se o teste KMO de medida de adequação da amostra. O resultado expresso neste teste $(0,801)$ demonstra um bom grau de ajuste para aplicação da técnica Análise Fatorial Exploratória, sendo ratificado pelo teste Bartlett de Esfericidade ao nível de significância 0,000.

Foram encontrados cinco fatores que explicam $67,65 \%$ da variância total. Esses fatores extraídos na análise foram rotacionados pelo método de rotação Varimax. Os resultados podem ser observados na Tabela 4. 
Tabela 4 - Matriz de componente rotativa

\begin{tabular}{|c|c|c|c|c|c|}
\hline \multirow[b]{2}{*}{ Variável } & \multicolumn{5}{|c|}{ Fatores } \\
\hline & 1 & 2 & 3 & 4 & 5 \\
\hline Variavel01 & ,841 &,- 152 & ,046 & 146 & 071 \\
\hline VariavelO2 &, 741 & 148 & ,338 & 206 & ,020 \\
\hline VariavelO3 & ,594 & 487 &,- 004 & ,046 &,- 056 \\
\hline VariavelO4 & ,715 & , 135 & , 172 & 398 & ,043 \\
\hline VariavelP5 & ,174 & ,666 & ,296 &,- 170 & ,023 \\
\hline VariavelP2 &,- 171 &, 646 & ,200 & ,328 & 110 \\
\hline VariavelO7 & ,230 &, 664 & ,017 & , 137 &,- 021 \\
\hline VariavelT3 &,- 081 & ,675 & ,112 & ,034 & 288 \\
\hline VariavelP1 & , 184 & ,024 & ,733 & ,005 & 149 \\
\hline VariavelP3 & , 051 & 148 & ,783 & ,113 &,- 067 \\
\hline VariavelP4 & , 100 & ,251 & ,714 & 167 & , 127 \\
\hline VariavelO5 & ,314 & 149 & , 079 &, 860 & ,034 \\
\hline VariavelO6 & ,247 & ,032 & , 161 & ,859 & 132 \\
\hline VariavelT1 &,- 005 &,- 003 & ,001 & 115 & ,863 \\
\hline VariavelT2 & , 086 & ,250 & 173 & ,024 & ,795 \\
\hline
\end{tabular}

Fonte: Dados da pesquisa (2015).

Para rodar a AFC no SmartPLS, a cada fator identificado na AFE (ou variável latente) foram atribuídos seus itens e entre os fatores foram incluídas setas estruturais de modo que todos estivessem conectados com todos (BROWN, 2006) e o esquema de ponderação para o algoritmo PLS (Partial Least Squares) foi o "Factor weightinh scheme" (HAIR JR. et al., 2014). Todas as variáveis tiveram carga fatorial acima de 0,5 em seus respectivos fatores. A Tabela 5 ilustra esta etapa da análise.

Tabela 5 - Cargas fatoriais das variáveis

\begin{tabular}{l|c|c|c|c|c}
\hline $\begin{array}{c}\text { Variáveis } \\
\text { / Fatores }\end{array}$ & $\begin{array}{c}\text { Fator 1 - } \\
\text { Organizaç } \\
\text { ão }\end{array}$ & $\begin{array}{c}\text { Fator 2 - Redes e } \\
\text { recompensas }\end{array}$ & $\begin{array}{c}\text { Fator 3 - } \\
\text { Característ } \\
\text { ica PVE }\end{array}$ & $\begin{array}{c}\text { Fator 4 - } \\
\text { Liderança }\end{array}$ & $\begin{array}{c}\text { Fator 5 - } \\
\text { Tecnologia }\end{array}$ \\
\hline VariavelO1 & 0,724 & & & & \\
\hline VariavelO2 & 0,869 & & & & \\
\hline VariavelO3 & 0,665 & & & & \\
\hline VariavelO4 & 0,874 & & & & \\
\hline VariavelO5 & & & & 0,952 & \\
\hline VariavelO6 & & & & & \\
\hline VariavelO7 & & 0,687 & & & \\
\hline
\end{tabular}


Determinantes do compartilhamento do conhecimento visando a cooperação internacional em ciência e tecnologia no Brasil
Fernanda Cristina Barbosa Pereira Queiroz; Helena de Fátima Nunes Silva; Paulo Henrique de Almeida

\begin{tabular}{l|r|r|r|r}
\hline VariavelP5 & 0,727 & & & \\
\hline VariavelP2 & 0,735 & & & \\
\hline VariavelT3 & 0,693 & & & \\
\hline VariavelP3 & & 0,765 & & \\
\hline VariavelP4 & & 0,860 & & \\
\hline VariavelP1 & & 0,721 & & \\
\hline VariavelT1 & & & & 0,726 \\
\hline VariavelT2 & & & & 0,950 \\
\hline
\end{tabular}

Fonte: Dados da pesquisa(2015)..

Na Tabela 6 observa-se que todos os construtos estão com variância média extraída (Average Variance Extracted - AVE) acima de $50 \%$, alfa de Cronbach acima de 0,6 e confiabilidade composta (composite reliability) acima de 0,8, assegurando a aceitação das variáveis nos fatores. Os cinco (5) fatores encontrados na AFE e validados na AFC foram denominados de Organização Receptora, Redes e Recompensas, Características do Transmissor, Gestão e Tecnologia.

Tabela 6 - Indicadores selecionados

\begin{tabular}{|c|c|c|c|c|c|}
\hline Fatores & Variáveis & $\begin{array}{c}\% \\
\text { Variância } \\
\end{array}$ & $\begin{array}{c}\text { Cronbachs } \\
\text { Alpha }\end{array}$ & $\begin{array}{l}\text { Composite } \\
\text { Reliability }\end{array}$ & AVE \\
\hline \multirow{4}{*}{$\begin{array}{l}\text { Fator } 1 \text { - } \\
\text { Organização } \\
\text { Receptora }\end{array}$} & Infraestrutura & \multirow{4}{*}{29,963} & \multirow{4}{*}{0,786} & \multirow{4}{*}{0,866} & \multirow{4}{*}{0,622} \\
\hline & Clima & & & & \\
\hline & Cultura & & & & \\
\hline & Estrutura & & & & \\
\hline \multirow{4}{*}{$\begin{array}{l}\text { Fator } 2 \text { - } \\
\text { Redes e } \\
\text { recompensas }\end{array}$} & $\begin{array}{l}\text { Redes de } \\
\text { relacionamento e } \\
\text { cooperação }\end{array}$ & \multirow{4}{*}{43,378} & \multirow{4}{*}{0.674} & \multirow{4}{*}{0,803} & \multirow{4}{*}{0,505} \\
\hline & Publicação & & & & \\
\hline & $\begin{array}{l}\text { Recompensas e } \\
\text { incentivos }\end{array}$ & & & & \\
\hline & $\begin{array}{l}\text { Redes sociais e } \\
\text { aplicativos }\end{array}$ & & & & \\
\hline \multirow{3}{*}{$\begin{array}{l}\text { Fator } 3 \text { - } \\
\text { Característica } \\
\text { do } \\
\text { Transmissor }\end{array}$} & $\begin{array}{l}\text { Habilidade de } \\
\text { Comunicação }\end{array}$ & \multirow{3}{*}{52,560} & \multirow{3}{*}{0.687} & \multirow{3}{*}{0,827} & \multirow{3}{*}{0,615} \\
\hline & Confiança & & & & \\
\hline & Experiência & & & & \\
\hline \multirow{2}{*}{$\begin{array}{l}\text { Fator } 4 \text { - } \\
\text { Gestão }\end{array}$} & Alta direção & \multirow{2}{*}{60,748} & \multirow{2}{*}{0.889} & \multirow{2}{*}{0,947} & \multirow{2}{*}{0,900} \\
\hline & Liderança & & & & \\
\hline \multirow{2}{*}{$\begin{array}{l}\text { Fator } 5 \text { - } \\
\text { Tecnologia }\end{array}$} & Internet & \multirow{2}{*}{67,658} & \multirow{2}{*}{0.645} & \multirow{2}{*}{0,832} & \multirow{2}{*}{0,715} \\
\hline & Sistemas de Informação & & & & \\
\hline
\end{tabular}

Fonte: Dados da pesquisa (2015).

Os fatores que influenciam o compartilhamento de informações e conhecimento entre os pesquisadores estrangeiros e brasileiros vinculados às Instituições de Ensino e Pesquisa nacionais podem ser divididos em cinco tipos. No contexto organizacional, o fator Organização Receptora agrupa as variáveis infraestrutura, cultura, clima e estrutura 
organizacional e estão relacionados a aspectos da instituição do proponente que recebeu o PVE. Por sua vez, o fator Gestão compreende as variáveis Liderança e alta administração.

As características da equipe, medida pelo fator Redes e Recompensas inclui as variáveis redes de relacionamento e cooperação, publicações, recompensas e incentivos e a variável redes sociais e aplicativos. Este fator indica a importância das redes presenciais e/ou virtuais e os resultados esperados (recompensas) para 0 compartilhamento. O fator Características do Transmissor, compreende aspectos interpessoais, e consiste no agrupamento de variáveis habilidade de comunicação, confiança e experiência. E o fator Tecnologia agrupou as variáveis Internet e Sistemas de Informação.

\section{Considerações Finais}

Os fatores encontrados que influenciam o compartilhamento de informações e conhecimento entre os pesquisadores estrangeiros e brasileiros sintetizaram as variáveis relacionadas às características das instituições de ensino e pesquisa que receberam os PVE, às características do PVE e à tecnologia. Tem-se, portanto, que fatores pessoais, relacionados às características do transmissor e possibilidade de recompensas e formação de redes, fatores organizacionais referentes a organização receptora e gestão, e fatores tecnológicos são determinantes para o compartilhamento do conhecimento entre os proponentes e os PVE.

A confiança, a tecnologia da informação (internet e sistemas de informação), experiência e habilidade de comunicação do pesquisador estrangeiro foram aspectos que apresentaram as médias mais altas e pode-se inferir que possibilitam o compartilhamento do conhecimento entre os componentes da amostra investigada.

O Programa Ciência sem Fronteiras segue a tendência internacional de cooperação e interação de pesquisadores. O objetivo principal é o desenvolvimento da ciência e da tecnologia no país. Neste sentido, observou-se que 0 incremento do trabalho cooperativo tem-se evidenciado pelo menos a partir de 1994 (LONGO; OLIVEIRA, 2000), como atestam iniciativas do Ministério da Ciência e Tecnologia (MCT/CNPq).

Acredita-se que as pesquisas cooperativas, além de movimentarem recursos de diferentes naturezas, possibilitam relações sociais importantes. Essas relações fomentam pesquisas e tecnologias que são efetivadas pelos resultados de publicações em co-autoria, aprendizagem mútua e inovação. Assim, ao estabelecer os fatores que favorecem 0 compartilhamento de conhecimento, indica-se a ponta do iceberg no processo de desenvolvimento da ciência e da tecnologia. Este estudo pode inspirar outras pesquisas sobre os desdobramentos do compartilhamento do conhecimento entre pesquisadores nacionais e estrangeiros.

Para trabalhos futuros, sugere-se ampliar o tamanho da amostra incluindo os proponentes dos projetos aprovados em 2014 e 2015 e 
também com os PVE. Outra possibilidade de pesquisa é a análise quantitativa e qualitativa da produção conjunta dos pesquisadores e os seus impactos no desenvolvimento científico e tecnológico do país.

\section{Referências}

AJMAL, M. M.; KOSKINEN, K. U. Knowledge transfer in project-based organizations: an organizational culture perspective. Project Management Journal, v. 39, n. 1 p. 7-15, 2007.

ALCARÁ, A. R. et al. Fatores que influenciam o compartilhamento da informação e do conhecimento. Perspectivas em Ciência da Informação, v. 14, n. 1, p. 170-191, 2009.

BARON, R. A.; MARKMAN, G. D. Beyond social capital: how social skills can enhance entrepreneurs. The Academy of Management Executive, $\mathrm{v}$. 14, n. 1, p. 106-16, 2000.

BOUSARI, R.G; HASSANZADEH, M. Factors that affect scientists' behavior to share scientific knowledge. Collnet Journal of Scientometrics and Information Management, Istanbul, v. 6, n. 2, p 215-227, 2012.

BRASIL. Ministério da Ciência, Tecnologia e Inovação. Ministério da Educação. Programa Ciência Sem Fronteiras. Brasília, 2011.

BROWN, T. A. Confirmatory factor analysis for applied research. New York: Guilford Press, 2006.

BRUNI, L. Comunhão e as novas palavras em economia. São Paulo, SP: Cidade Nova, 2005.

CARVALHO, I. M.; MENDES, S. P.; VERAS, V. M. Gestão do conhecimento: uma estratégia empresarial. Brasília: J. J. Gráfica e Comunicações, 2006. Disponível em: <http://www.serpro.gov.br>. Acesso em: 9 nov. 2016.

CHASE, R. The knowledge-based organization: an international survey. Journal of Knowledge Management, v. 1 n. 1, p. 38-49, 1997.

CYR, S; CHOO, C. W. The individual and social dynamics of knowledge sharing: an exploratory study. Journal of Documentation, v. 66, n. 6, p. 824-846, 2010.

CHO, N.; LI, G.; SU, Ch. An empirical study on the effect of individual factors on knowledge sharing by knowledge type. Journal of Global Business and Technology, v. 3, n. 2, p. 1-15, 2007.

DAVENPORT, T. H.; PRUSAK, L. Working knowledge: how organisations manage what they know. Boston: HBS Press, 1998.

DE LONG, D.; FAHEY, L. Diagnosing cultural barriers to knowledge management. Academy of Management Executive, v. 14, n. 4, p. 113$127,2000$.

DELGADO, A. S. et al. Fatores que influenciam no processo de compartilhar conhecimento em um projeto de extensão: um estudo do 
Projeto Chiquitos, na Chiquitania, Boliviana. In: COLÓQUIO DE GESTÃO UNIVERSITÁRIA EN AMERICAS, 13. Buenos Aires, 27-29 nov. 2013. Anais... Buenos Aires: UFSC; Universidade Mar Del Plata, 2013. p. 1-19.

DUARTE, R. P. Cooperação internacional para o desenvolvimento em ciência e tecnologia: a participação brasileira na Organização Européia para pesquisa nuclear (CERN). Journal of Technology Management \& Innovation, v. 3, n. 4, p. 133-151, Dec. 2008. Disponível em: $<$ http://www.jotmi.org/index.php/GT/article/view/cas17/468>. Acesso em: 2 dez. 2015.

DUGUID, P. The art of knowing: social and tacit dimensions of knowledge and the limitsof the community of practice. The Information Society, $\mathrm{v}$. 21, p. 109-18, 2005.

ENSIGN, P. C. Knowledge sharing among scientists: why reputation matters for R\&D in multinational firms. New York: Palgrave Macmilan, 2008.

ENSIGN, P. C.; HEBERT, L. How reputation affects knowledge sharing among colleagues. MIT Sloan Management Review, v. 51, n. 2, p. 79-81, 2010.

FUNDAÇÃO DE AMPARO À PESQUISA DO ESTADO DE SÃO PAULO. Atração de talentos. Revista Pesquisa FAPESP. 2010. Disponível em: <http://revistapesquisa.fapesp.br/2010/09/02/atração-de-talentos/>.

Acesso em: 24 ago. 2013.

FLORIANO, P. R. Os sete obstáculos ao compartilhamento do conhecimento e três maneiras de superá-los. 2010. Disponível em: <http://www.terraforum.com.br/biblioteca/Documents/libdoc00000179v0 02Sete\%20obstaculos\%20ao\%20compartilhamento\%20do\%20con.pdf>.

Acesso em: 6 ago. 2014

HAIR, J. F. et al. A primer on partial least squares structural equation modeling. Sage: Thousand Oaks, 2014

HO, L.-A.; KUO, T.-H.; LIN, B. How social identification and trust influence organizational online knowledge sharing. Internet Research, v. 22 n. 1, p. 4-28, 2012.

HOLSTE,J. S.; FIELDS D. Trust and tacit knowledge sharing and use. Journal of Knowledge Management, v. 14, n. 1, p. 128-140, 2010.

HONG, D.; SUH, E.; KOO, C. Developing strategies for overcoming barriers to knowledge sharing based on conversational knowledge management: A case study of a financial company. Expert Systems with Applications, n. 38, p. 14417-14427, 2011.

INGRAM, P.; BAUM, J. A. C. Opportunity and constraint: organizations learning from the operating and competitive experience of industries, Strategic Management Journal, v. 18, special Summer issue, p. 75-98, 1997. 
IPE, M. Knowledge sharing in organizations: a conceptual framework. Human Resource Development Review, v. 2, p. 337-359, 2003.

JO, S. J.; JOO, B-K. Knowledge sharing: the influences of learning organization culture, organizational commitment, and organizational citizenship behaviors. Journal of Leadership \& Organizational Studies, v. 18 , n. 3, p. 353-364, 2011.

KIM, S.; LEE, H. The impact of organizational context and information technology on employee knowledge-sharing capabilities. Public Administration Review, v. 66, n. 3, p. 370-385, 2006.

KNOCKAERT, M. et al. The relationship between knowledge transfer, top management team composition, and performance: the case of Sciencebased entrepreneurial firms. Entrepreneurship: theory and practice, v. 35, n. 4., p. 777-803, Jul 2011.

CLARYSSE, B. et al. The relationship between knowledge transfer, top management team composition, and performance: the case of sciencebased entrepreneurial firms. Entrepreneurship Theory and Practice, v. 35, n. 4, p. 777-803, 2011.

KUO, T-H. How expected benefit and trust influence knowledge sharing, Industrial Management \& Data Systems, v. 113, n. 4, p. 506-522, 2013.

LIN, H.; LEE, G. G. Effects of socio-technical factors on organizational intention to encourage knowledge sharing. Management Decisions, v. 44, n. 1 , p. 74-88, 2006.

LIN, H. F. Knowledge sharing and firm innovation capability: an empirical study. International Journal of Manpower, v. 28, n. 3/4, p. 315-32, 2007.

LONGO, W. P.; OLIVEIRA, A. R. P. de. Pesquisa cooperativa e centros de excelência. Parcerias Estratégicas, Brasília, n. 9, p. 129-144, 2000.

LUCAS, L. The impact of trust and reputation on the transfer of best practices. Journal of Knowledge Management, v. 9, n. 4, p. 87-101, 2005.

MANSFIELD, E. Academic research and industrial innovation. Research Policy, v. 20, n. 1, p. 1-12, 1991.

NAHAPIET, J.; GHOSHAL, S. Social capital, intellectual capital, and the organizational advantage. Academy of Management Review, v. 23 n. 2, p. 242-66, 1998.

NODARI, F. et al. Facilitadores e barreiras para o compartilhamento de conhecimento em cadeias de suprimentos. Produto \& Produção, v. 13, n. 3, p. 49-62, out. 2012.

NONAKA, I.; TAKEUCHI, H. Criação de conhecimento na empresa: como as empresas japonesas geram a dinâmica da inovação. 14. ed. Rio de Janeiro: Campus, 1997. 
ORDAZ, C. C.; CRUZ, J. G.; GINEL, E. S. Facilitadores de los procesos de compartir conocimiento y su influencia sobre la innovación. Cuadernos de Economía y Dirección de la Empresa, n. 42, p. 113-150, mar. 2010.

OSINSKI, M.; DARLAN JOSÉ ROMAN, D. J.; SELIG, P. M. Compartilhamento de conhecimento: estudo bibliométrico das publicações acadêmicas realizadas de 1994 a 2014. Perspectivas em Ciência da Informação, v. 20, n. 4, p. 149-162, dez. 2015.

PAGHALED, M.; SHAFIEZADEH, E.; MOHAMMADI, M. Information technology and its deficiencies in sharing organizational knowledge. International Journal of Business and Social Science, v. 2, n. 8, p. 192198, Mai 2011.

PHILLIPS, K. W. et al. Diverse groups and information sharing: the effects of congruent ties. Journal of Experimental Social Psychology, n. 40, p. 497-510, 2004.

RAPINI, M. S; RIGHI, H. M. Interação universidade-empresa no Brasil em 2002 e 2004: uma aproximação a partir dos grupos de pesquisa do CNPq. In: ENCONTRO NACIONAL DE ECONOMIA POLITICA, 10., Natal, 2005. Anais... Natal: UFRN; ANPEC, 2005.

RIEGE, A. Three-dozen knowledge-sharing barriers managers must consider. Journal of Knowledge Management, v. 9, n. 3, p. 18-35, 2005.

SATO, K. A. S; SILVA, H. N.; DRAGO, I. A gestão do conhecimento sob a perspectiva das sete dimensões: o caso do projeto perfis profissionais para o futuro da indústria. Inf. Inf., Londrina, v. 18, n. 1, p. 142-168, jan./abr. 2013.

TOHIDINIA, Z.; MOSAKHANI, M. Knowledge sharing behaviour and its predictors. Industrial Management + Data Systems, v. 110, n. 4, p. 611$631,2010$.

WANG, S.; NOE, R. A. Knowledge sharing: a review and directions for future research. Human Resource Management Review, v.20, n. 2, p. $115-131,2010$.

WANG, S.; NOE, A. R.; WANG, Z.-. Motivating knowledge sharing in knowledge management systems: a quasi field experiment. Journal of Management, v.25, n.3, p. 978-1009, may, 2014.

WESTPHAL, A. M. S. Egresso da primeira chamada do Programa Ciência sem Fronteiras: reflexos no sistema educacional brasileiro (Learning with outcomes). 120f. 2014. Dissertação (Mestrado em Educação) Universidade Católica de Brasília, Brasília, 2014.

WOLFE, Ch.; LORAAS, T. Knowledge sharing: the effects of incentives, environment, and person. Journal of Information Systems, v. 22, n. 2, p. 53-76, 2008. 